\title{
El desafío de construir una Psicología del desarrollo crítica en sociedades inhóspitas \\ The Challenge of Constructing a Critical Developmental Psychology in Inhospitable Societies
}

\author{
Carlos Iván Orellana \\ Universidad Centroamericana José Simeón Cañas, El Salvador
}

\begin{abstract}
Resumen
La Psicología del desarrollo constituye un campo de conocimiento cuya existencia se da por descontada en el imaginario académico de la psicología salvadoreña. No obstante, existe una Psicología del desarrollo dominante que se ve reproducida en textos, clases y discursos, mientras que otra distinta, consecuente con la realidad histórica donde su estudio tiene lugar, aún es una tarea pendiente. Al considerar la descontextualización como uno de los presupuestos fundamentales de la Psicología del desarrollo dominante al uso, en este artículo de carácter teórico se reflexiona sobre esta desde la perspectiva del caso salvadoreño en tanto sociedad inhóspita: aquella que compromete el avance del ciclo vital de la mayoría de la población por, entre otras características, ser violenta y desigual. Son sometidas a escrutinio las conocidas categorías analíticas de la Psicología del desarrollo definidas como influencias normativas y no normativas para mostrar la relatividad y los sesgos de su aplicación usual. Se sostiene que, en el marco de una sociedad inhóspita, una Psicología del desarrollo debe considerar, al menos, procesos de adultización prematura en la niñez y la juventud, la improbabilidad de biografías estables, la vejez precaria así como las características típicas que muestran los miembros del contexto de interés. Se propone avanzar a una Psicología del desarrollo crítica: la necesidad de reacomodar su quehacer y reinventarse epistemológicamente para construir un conocimiento situado, propio y multidisciplinario que supere cegueras, provincianismos y reduccionismos académicos.
\end{abstract}

Palabras clave: sociedad inhóspita, Psicología del Desarrollo, Psicología del Ciclo Vital, Psicología Crítica, influencias normativas y no normativas, ideología, epistemología

Carlos Iván Orellana, Departamento de Psicología y de Salud Pública, Universidad Centroamericana José Simeón Cañas, El Salvador.

La correspondencia en relación con este artículo se dirige a Carlos Iván Orellana, dirección electrónica: psi.ciorellana@gmail.com 


\title{
C. Orellana
}

\begin{abstract}
Developmental Psychology is a field of knowledge whose existence is taken for granted in the academic imagination of Salvadoran psychology. However, there is a mainstream Developmental Psychology that is reproduced in texts, lectures and discourses, while a different one, consistent with the historical reality where its study takes place, and is still a pending task. Considering de-contextualization as one of its fundamental assumptions, this theoretical article deals with the situation of Developmental Psychology from the perspective of the Salvadoran case as an Inhospitable Society: one that distorts the progress of most of its people's life cycle, due to, among other features, its violence and social inequality. Very well known analytical categories such as normative and non-normative developmental trends are subjected to scrutiny to show the relativity and biases of their usual applications. It is argued that, in the context of an inhospitable society, Developmental Psychology should consider at least: Early Childhood and Youth Adultisation Processes, the improbability of stable biographies, Precarious Old Age and the typical features of individuals living in the context of interest. It aims to advance a Critical Developmental Psychology: the need to rearrange the work and to reinvent itself epistemologically in order to construct a situated, appropriated and multidisciplinary knowledge that overcomes blindness, provincialism and academic reductionism.
\end{abstract}

Keywords: Inhospitable Society, Developmental Psychology, Psychology of the Life Span, Critical Psychology, Normative and Non-Normative Developmental Trends, Ideology, Epistemology

\section{Inexistencia y apropiación de un campo de conocimiento}

La experiencia de estudio y de práctica docente sobre la Psicología del desarrollo en El Salvador permite arribar a dos conclusiones básicas y entretejidas entre sí: la primera consiste en que la Psicología del desarrollo psicológico humano no existe en el país; que no es lo mismo que decir que no se conozca, que no se estudie o que no se encuentren textos disponibles sobre la materia. La segunda conclusión, inevitable deriva de la anterior, implica que la Psicología del desarrollo al uso, la que se encuentra accesible en los conocidos manuales de la materia, retrata el desarrollo humano y sus hitos principales bajo coordenadas analíticas bastante ajenas a la realidad de la población salvadoreña.

El primer aspecto, la inexistencia académica del campo de estudio, queda constatado por los vacíos que deja el menor esfuerzo de búsqueda al respecto: no existen estudios sistemáticos, publicaciones (artículos, y menos aún revistas o libros sobre el tema) o instituciones u organismos centrados en el estudio o la investigación del ciclo vital y sus avatares. Diferente es que, en el país, cada tanto, se llevan a cabo estudios que ineludiblemente involucran o se refieren a categorías sociales o a individuos situados en alguna etapa del desarrollo humano. En el último tiempo, es el caso de publicaciones sobre niñez a propósito del vigente y masivo fenómeno de la migración irregular (Gaborit, Zetino, Orellana, \& Brioso, 2015; Gaborit et al., 2016), encuestas generales sobre juventud (Santacruz \& Carranza, 2009), estudios sobre factores de riesgo y de protección de violencia en jóvenes (Gutiérrez, 2013) o sobre la creciente importancia y las características sociodemográficas de los adultos mayores en el país (Córdova, Burgos, Tablas, \& Rodríguez, 2010). Sin embargo, si comprendemos la Psicología del desarrollo como "el estudio científico de los procesos sistemáticos de cambio y de estabilidad de las personas" (Papalia y Feldman, 2012, p.4), no se trata de aproximaciones cuya dirección sea explorar, con propósitos básicos o aplicados, el ciclo vital de los individuos de una sociedad desde la óptica 
específica de la Psicología del desarrollo (como contraste se sugiere ver pormenores del caso costarricense en Tapia-Valladares, Carmiol-Barbosa, \& Rosabal-Coto, 2012).

Afirmar que tal rama de la Psicología no existe en el país y podría parecer una afirmación demasiado contundente para algunos. En realidad, las señales de vida solo se verifican a partir de un confinamiento epistémico: el campo de la Psicología del desarrollo es identificable, sí, pero fundamentalmente en el particular acervo de mayor o menor conocimiento del estudiante o del profesional que echa mano de las herramientas que la Psicología del desarrollo ofrece; en la reedición interminable de manuales internacionales traducidos al castellano por distintas casas editoriales extranjeras; o a través de una incipiente institucionalización circunscrita al típico curso que ofertan los programas de estudio de grado en las universidades ${ }^{1}$. La interrogante que se deprende de las constataciones anteriores es inevitable: ¿de qué conocimiento disponible se han servido y se sirven tales profesionales o tales cursos de formación superior en ausencia de una producción contextualizada de conocimiento? La respuesta a esta pregunta nos sitúa frente al segundo aspecto que aquí se está discutiendo: la alienación de los contenidos de la Psicología del desarrollo mainstream con respecto a las características del desarrollo humano propias del contexto desde el cual se estudia (en este caso, y especialmente, cuando se trata de un país como El Salvador).

La respuesta a la interrogante anterior es que en virtud del reciclaje histórico de contenidos y la disponibilidad de textos sobre la materia, en las aulas universitarias como en el conocimiento de estudiantes y profesionales, circulan -por supuesto- los autores, las teorías y los enfoques esperados (Piaget, Erikson, Vygotsky, Baltes, Kübler-Ross...). Se trata de un conocimiento apropiado, en un doble sentido: en cuanto que se trata del conocimiento "correcto" y dominante, y en tanto que un conocimiento que ha sido tomado o hecho propio, sin que constituya una propuesta original ni siempre adecuada. Sigue siendo válida la advertencia que hace ya treinta años se hacía por estos lados, a propósito de la dependencia de la Psicología de nuestros países, respecto a la Psicología norteamericana: aún necesita la respiración asistida que ofrece el mimetismo cientista (MartínBaró, 1986), tendencia que la lleva a copiar teorías y modelos foráneos antes que a producir lo propios. Estamos ante prácticas de objetivación (Ibáñez, 2001) o procesos de búsqueda de un objeto que, sin embargo, eluden la construcción de un objeto de estudio propio para agenciarse un conocimiento producido de forma apriorística.

Los riesgos de esta práctica no se limitan al uso y la reproducción descontextualizada del conocimiento o a la ilusión de prestigio académico que conlleva ataviarse con marcos conceptuales reconocidos. De fondo, el empleo acrítico y sistemático de conocimiento dominante pero ahistorizado, consolida subrepticiamente tanto distorsiones ideológico-epistemológicas como modelos prescriptivos para el análisis y el estudio del ciclo vital de las personas concretas, así como de la forma particular en que tal proceso se supone que debe ser vivido. Por eso, es tan revelador para el aprendizaje de las y los estudiantes cuando, en las clases de esta materia, se exponen textos de Psicología del desarrollo cuyas descripciones o ilustraciones muestran niños y niñas que, si mueren, lo hacen por causas naturales o que siempre están escolarizados. Donde se afirma con respecto al embarazo adolescente que las políticas públicas promueven el uso de anticonceptivos, que las adolescentes pueden decidir abortar (mientras

\footnotetext{
${ }^{1}$ Según Calderón de Orellana (2009), la Psicología del desarrollo en El Salvador tendría algo más de medio siglo de andadura universitaria pues aparece muy pronto (1959), bajo la rúbrica de Psicología Evolutiva, en las reformas de los planes de estudio de la carrera de Psicología de la Universidad Nacional. Precisamente, parte de lo que aquí se está cuestionando es que el grado de desarrollo y construcción de la Psicología del desarrollo no se encuentra acorde con su rancia existencia en los recintos universitarios del país. Sin embargo, por estos lados, esta última afirmación podría aplicar para casi todas las ramas del quehacer de la Psicología.
} 


\section{Orellana}

el padre adolescente queda casi ausente o implícito en las explicaciones), o se llega a afirmar que los hombres anhelan la capacidad de parir de las mujeres. Son textos donde la crisis de madurez masculina se ilustra con el paseo en una moto Harley-Davidson, donde el climaterio femenino se reduce a manejables cambios biológicos y mentales aislados de las presiones culturales o donde la jubilación se ve representada por un hombre con mirada triunfal en la proa de su yate. Asimismo, si partimos de la generalidad de la retórica expositiva de los textos al uso de la disciplina (algunos se mencionan a continuación), es inevitable corroborar el sesgo positivista-cuantitativo y heteronormativo de sus perspectivas o la recurrente construcción de modelos teóricos a partir de etapas que, en la práctica, más responden a las consabidas advertencias de que no funcionan como el mismo modelo plantea.

Los señalamientos anteriores están en consonancia con las opiniones de Burman $(1997,2015)$ acerca de la Psicología del Desarrollo mainstream. Según la autora, la Psicología del desarrollo dominante conlleva, entre otras cosas, presupuestos socioculturales conservadores; concepciones individualistas del desarrollo; incurre en biologismos y construcciones abstraccionistas "promedio" alejadas de individuos y de contextos reales; emplea convenientes eufemismos que dibujan el desarrollo como un proceso "natural", lineal, progresivo y estable; y, en general, hace caso omiso de las relaciones de poder (e.g. género) que se despliegan en sus teorías y métodos. Por todo ello, conviene reconocer que lo que se suele estudiar, replicar y "saber" por estos lados a la hora de aproximarse a la psicología del desarrollo humano dista de ser un modelo universal. Se trata, en realidad, del estilo de vida de una clase media, fundamentalmente blanca, proveniente de un país desarrollado, cuya caracterización se ve arropada con los engañosamente asépticos presupuestos ideológicos mencionados. Bajo estas condiciones, y en contraste, el ciclo vital de un salvadoreño o una salvadoreña "promedio" constituye y se presenta como una especie de anomalía existencial y epistemológica.

\section{La excepción como norma en sociedades inhóspitas}

Para visibilizar mejor lo que aquí se argumenta, sirve problematizar un recurso analítico usual de la Psicología del desarrollo: los denominados procesos normativos. Precisamente, a partir de una revisión de manuales de la materia al uso (Arranz-Freijo, 1998; Craig, 2001; Feldman, 2007; Fernández-Lópiz, 2000; Lefrancois, 2001; Papalia \& Feldman, 2012; Rice, 1997; Shaffer \& Kipp, 2010), podemos convenir que los patrones o influencias normativas o normadas conforman el objeto de interés de la Psicología del desarrollo o que, al menos, sugieren la existencia de acontecimientos "esperables" o "típicos", que deberían ocurrir a los individuos, bajo la presuposición del mantenimiento de todas las condiciones constantes. Más allá de la artificialidad de toda clasificación, tales patrones suelen dividirse, primero, en procesos normados por la edad o por la historia. Serían procesos normativos relativos a la edad los siguientes: niñez, infancia, adolescencia, juventud, adultez y vejez; adquisición de pensamiento concreto y posterior desarrollo de pensamiento abstracto; proceso educativo formal, trabajo y jubilación; noviazgo, consolidación de pareja, matrimonio, viudez, entre otros. Mientras que se suelen mencionar como ejemplos de procesos normados por la historia aspectos que afectan a cohortes enteras: guerras, prosperidad, crisis o alguna epidemia; es decir se trataría de secuencias que, según la Psicología del desarrollo dominante, de manera respectiva, responden a la lógica de un desarrollo esperable, apropiado, adaptado y lógico, o a circunstancias inescapables debido a su alcance masivo o su imprevisibilidad. Lo contrario sería un patrón no normativo, ideográfico o idiosincrático; o sea, experimentar circunstancias "esperables" en momentos inesperados (e.g. estudiar educación primaria en la adultez), vivir eventos atípicos (e.g. un desastre o un accidente) o propiciar antinormatividad en la propia vida por voluntad propia (e.g. tener hijos en la vejez o renunciar a un empleo estable para dedicarse a recorrer el mundo haciendo autostop en las carreteras). 
La revisión de las categorías de normatividad y de no normatividad permite identificar una tensión analítica referida a dos aspectos relevantes: primero, los procesos normativos de la edad resultan más usuales y más sencillos para el discurso dominante de la Psicología del Desarrollo, porque se amparan en realidades biológicas e individuales, bastante menos invariables. En segundo lugar, por lo general, la retórica común de los textos al uso manifiesta una lectura ambigua o imprecisa cuando refieren a procesos normativos asociados a la historia y cuando se refieren a los procesos no normativos. Dicho de otra forma: la Psicología del desarrollo parece encontrar los cimientos de su narrativa principal al prestar atención a realidades más o menos inamovibles, pero parece que le sudan las manos y que tartamudea a la hora de intentar aprehender aspectos colectivos o contextuales que afectan el supuestamente lineal desarrollo psicológico humano. $\mathrm{O}$, acaso, ¿podemos aceptar que una guerra o los despidos masivos acaecidos en el marco de la crisis económica de los últimos años, por afectar a muchos, constituyen sin más una "influencia normativa"? ¿Qué pasa con el carácter "adaptativo" o "desadaptativo" de los procesos con independencia de que los mismos sean normativos o no normativos? Por ejemplo, ¿no sería más preciso convenir que pueden existir influencias normativas desadaptativas que derivan en el socavamiento de cualquier noción de trayectoria vital lineal u homeostática y que pueden hacer proliferar problemas de distinta índole? Además, ¿será que las influencias no normativas siempre deben arrastrar una connotación negativa? ¿No cabe considerar que una influencia no normativa -una mudanza, un divorcio, retomar los estudios o ejercer y disfrutar la propia sexualidad a pesar del convencionalismo social que sostiene que "ya no se está en edad para ello" o cuando se desafía la presión mojigata y sancionadora de la propia cultura- pueda constituir una gesta emancipadora y hasta una expresión clara de salud mental?.

Según Rice (1997), los procesos normativos traen menos problemas a la gente. Esto sería así porque, se asume, no son repentinos, inesperados o contraproducentes. Cabría señalar que el estudio de los procesos normativos también trae menos problemas a quienes deciden escoger o no saben que han escogido estudiar tales procesos. Porque, ¿qué ocurre cuando la norma es precisamente la antinormatividad? ¿Qué decir cuándo la normatividad confunde el maltrato con la disciplina infantil, cuando cercena proyectos vitales o clausura horizontes de desarrollo? ¿Qué ocurre cuando el desarrollo humano cuenta con condiciones persistentemente inestables, regresivas y hasta violentas? La mención previa de algunos estudios nacionales que se concentran en alguna de las etapas del desarrollo no solo ofrece pistas del abordaje indirecto que reciben los temas de interés del campo. Dichos estudios también ponen de manifiesto que las etapas del desarrollo son de interés en El Salvador debido a amenazas que se ciernen sobre ellas (migración forzada, violencia o imposibilidad de lograr un retiro digno). Aun conviniendo en que hasta en la peor de las circunstancias humanas existen normas, es claro que constituye un planteamiento muy sesgado concebir como normativo el desarrollo biologista o bajo condiciones constantes, mientras que la circunstancia colectiva o las variaciones de contexto -aún consideradas como normativas-no parecen afectar dicho desarrollo lineal. No es casualidad tampoco que las influencias no normativas sean llamadas idiosincráticas o ideográficas, o que cada uno por su decisión pueda verse inmerso en alguna de ellas: la clave es

\footnotetext{
2 Debates sobre binomios como normalidad-anormalidad o individuo desadaptado-sociedad desadaptativa escapan al alcance de este escrito. Aquí, se está enfatizando que las fronteras entre categorías analíticas como estas conforman esfuerzos de aproximación, construcciones difusas, herramientas de enunciación limitadas y que, para el caso, en determinadas circunstancias, lo normativo puede ser contraproducente para el desarrollo humano como lo no normativo una tabla de salvación para la existencia. Asimismo, como será reiterado arriba, mucho del interés de este trabajo se sintetiza en considerar que, en ciertos contextos, lo normativo es precisamente lo antinormativo: lo que rehúye de la estabilidad, la regularidad, la capacidad de anticipación y de control de los individuos, con las implicaciones que eso trae para su ciclo vital y para quienes lo piensan como objeto de estudio.
} 


\section{Orellana}

denodadamente individualista. Como si, precisamente, lo no normativo siempre respondiese a la responsabilidad o a la culpa de un individuo excéntrico o a la mala fortuna de la vida que se ha cebado con él o con ella.

En contraposición al carácter ahistoricista propio de la Psicología del desarrollo dominante antes apuntado, es menester considerar lo que conlleva la construcción del campo de la Psicología del desarrollo en sociedades que podrían ser caracterizadas como inhóspitas. Una sociedad inhóspita sería aquella caracterizada por contar con facetas estructurales (economía, política, cultura, vínculos humanos, etc.) que comprometen de manera reiterada y constante el bienestar y hasta la supervivencia de la mayoría de sus miembros. Por ejemplo, podría decirse que en estas, en términos relacionales, predomina la violencia y la hostilidad; en términos institucionales y normativos, la laxitud, la debilidad y la desprotección; en materia socioeconómica son desiguales, mientras que culturalmente son retrógradas. Nada menos que sociedades fundamentalmente violentas, impunes, anómicas, precarias, incívicas, misóginas y chabacanas. No es una reflexión exagerada, alarmista o antojadiza. Desde el prisma de la desigualdad, en la misma dirección, apunta el trabajo de Pérez-Sáinz, Segura-Carmona y FernándezMontero (2012) al hablar de las sociedades fracturadas de Centroamérica: con excepción de Costa Rica y la zona urbana de Panamá, el resto de los países del istmo exhibiría altos niveles de exclusión social. Para estos autores, el caso salvadoreño, la sociedad inhóspita objeto de estas reflexiones, presenta rasgos tales como un bajo crecimiento económico, débil poder infraestructural del Estado, alta violencia social e inseguridad intensa, frágiles Estados de derecho y tendencia a enfrentar la violencia delictiva con más violencia, con medidas represivas.

Al reflexionar sobre la emergencia de la subjetividad migrante desde una perspectiva más sociopsicológica, en otra parte (Orellana, 2016) he propuesto la existencia de diadas de aspectos estructurales objetivos y subjetivos distintivos de las condiciones de existencia actuales de la generalidad de los salvadoreños: a) vulnerabilidad-precarización: la posibilidad constante de ir a peor y la experiencia reiterada de que ello está ocurriendo; b) amenaza-escenificación: la presencia de contingencias diversas que promueven la anticipación persistente y presente de calamidades futuras, $\mathrm{y}$ c) peligro-miedo: es decir, la vivencia de un contexto objetivamente violento que hace temer por perder la vida de forma igualmente violenta. Una sociedad, pues, inhóspita. En el marco de la discusión de la Psicología del desarrollo, este contexto conlleva la distorsión si no es que la obstaculización generalizada de los procesos normativos mencionados y, en general, la improbabilidad del avance estable del ciclo vital de las personas en sus distintas etapas, según su situación concreta en la estructura social

Estas son reflexiones insoslayables en las condiciones actuales en que se desenvuelven los acontecimientos, en un mundo globalizado y condicionado por una economía de mercado voraz que multiplica y complejiza los problemas que genera. Cualquier diagnóstico sobre las dificultades multifacéticas de la mayoría de países centroamericanos constituye, al final, una concreción cercana de condiciones mundiales bastante reconocibles. De ahí que la Psicología del desarrollo no puede pretender desarrollar su papel como campo de conocimiento científico y académico flotando en el cómodo vacío de cavilaciones importadas. Es a los oídos de esta Psicología a los que parecen dirigirse en particular las Naciones Unidas con su llamado a considerar "las

\footnotetext{
${ }^{3}$ Por si fuera necesario explicitarlo, aquí, se comprende una sociedad inhóspita como un tipo ideal, como una herramienta heurística para aproximarse a lo social que queda invisibilizado y fuera del análisis. Nunca se niega su heterogeneidad: en una sociedad inhóspita coexiste la miseria más humillante con la abundancia más suntuosa. Así, en ella, es posible identificar también ciclos vitales "de libro", armoniosos y estables. Sin embargo, eso es la excepción y no la norma. Antes bien, en El Salvador, la vida de la generalidad de las personas se ve sometida a los vaivenes inhóspitos que han sido expuestos con anterioridad y a condiciones de existencia que obligan a pensar de forma diferente el desarrollo humano, tal como se verá a continuación.
} 
capacidades asociadas con el ciclo de vida" (Programa de las Naciones Unidas para el Desarrollo [PNUD], 2014) o lo que es lo mismo, la imbricación contemporánea que se produce hoy entre el ciclo vital, sus hitos, sus tareas, etapas y transiciones en relación con circunstancias generalizadas de vulnerabilidad.

Una Psicología del desarrollo mínimamente situada y consecuente con realidades tan peculiares como la salvadoreña ${ }^{4}$, siempre debería considerar procesos y expresiones concretas atentatorias del desarrollo humano. Con base en diversas fuentes (Córdova et al., 2010; Dirección general de Estadística y Censos [DIGESTYC], 2014; Ministerio de Salud [MINSAL] e Instituto Nacional de Salud [INS], 2015; Orellana, 2012, 2014, 2016; Programa del Estado de la Nación, [PEN], 2011, 2016; PNUD, 2013), es posible proponer el siguiente inventario general y provisional de dichos aspectos:

a) Procesos de adultización prematura en la niñez y la juventud: mendicidad, trabajo infantil o colaboración en oficios varios en hogares y negocios en combinación o en lugar de cursar estudios formales; migración irregular de niños y de niñas; asunción de roles de cuido de otros niños y niñas o de adultos dependientes; conformación de hogares entre adolescentes; la naturalización cultural del abuso sexual, de la unión marital y de la maternidad de niñas y adolescentes; el suicidio de estas niñas y adolescentes ante el cierre vital que enfrentan; involucramiento en economías criminales y trayectorias delincuenciales.

b) Improbabilidad de biografias estables: desarrollo cognoscitivo, emocional y lingüístico dificultoso en la primera infancia debido a deficiencias en el cuido, la estimulación y la educación; problema de acceso, baja calidad o abandono de la escuela; incertidumbre ocupacional; informalidad laboral; empleo precario o trayectorias laborales inestables; muerte violenta, prematura y epidémica o desaparición de los progenitores y/o de sus hijos e hijas; multiplicación de la orfandad; viudez femenina prematura (y consiguiente erosión de condiciones materiales de existencia del núcleo familiar); familias desgarradas (por muerte violenta de uno o varios de sus miembros, por desaparición, por desarraigo debido a migración o por desplazamiento forzado debido a la situación de violencia; violencia doméstica).

c) Vejez precaria: ausencia de cobertura del sistema de pensiones; pensiones bajas; imposibilidad de retiro (necesidad de una ocupación remunerada o trabajo a perpetuidad) y trastoque o supresión del proceso psicosocial de la jubilación; decremento de esperanza de vida sana ${ }^{5}$; baja disponibilidad o inaccesibilidad a hogares de acogida; indigencia, abandono e incertidumbre existencial al final de la vida; muerte en soledad.

Las tres categorías previas deben ser consideradas como permeables y condicionantes entre sí (e.g. el embarazo adolescente o la muerte de los progenitores conllevaría adultización, compromete una biografía estable y podría traducirse a largo plazo en una vejez precaria). Otros aspectos complementarios y otras implicaciones son

\footnotetext{
${ }^{4}$ Después de todo, gran parte del istmo centroamericano, y especialmente los países que conforman el triángulo norte, presentan tales características peculiares (ver PEN, 2011). Como fue dicho antes, según el análisis de Pérez-Sáinz et al., (2012), solo Costa Rica y la zona urbana de Panamá, escapan a las implicaciones de constituir sociedades profundamente desiguales y excluyentes.

${ }^{5}$ Aumento de años de vida padeciendo enfermedades. Según el PEN $(2011,2016)$, en el último quinquenio la brecha que existe entre la esperanza de vida al nacer y la esperanza de vida sana en los adultos mayores salvadoreños supera los 10 años; es decir que, como tendencia general, cuando una persona supera los 60 años de edad en El Salvador, se abre para ella una ventana de morbilidad que puede prolongarse al menos unos once años. En Belice, este tiempo alcanzaría los 12 años y unos 10 años en promedio en el resto de Centroamérica. Además de la merma en la calidad de vida en la adultez tardía, esto conlleva una enorme presión para los sistemas de salud pública de los distintos países.
} 


\section{Orellana}

dignas de mención: el conservadurismo y la arraigada cultura religiosa que alimenta visiones heteronormativas de los vínculos humanos y la vigilancia atávica que se cierne sobre las mujeres, sus cuerpos y sus proyectos de vida. Asimismo, el oscurantismo que conlleva la clausura de la discusión social en torno a temas de salud sexual reproductiva, el aborto o la eutanasia. En medio de tanta muerte violenta, cabe reseñar, por último, la distorsión de procesos de duelo ante pérdidas constantes, súbitas, violentas y sin sentido de niños, niñas y jóvenes, individuos que no se suponía que debían fallecer en el momento, la forma y la frecuencia con que perdieron la vida.

Si algo de razón se lleva y la Psicología del desarrollo hegemónica reproduce el estilo de vida de la clase media de un país desarrollado, ¿cuál sería el perfil general de una persona salvadoreña promedio? Lefrancois (2001) nos recuerda que la Psicología del desarrollo trabaja con individuos y contextos promedio aunque, en cuanto que construcciones, tales individuos o contextos promedios, en realidad, no existen. De lo que no cabe duda es que si hubiera que hacer emerger un individuo concreto, dibujar el "tipo ideal" que podría ser el objeto de las reflexiones de una Psicología del desarrollo consecuente con la circunstancia histórica de un país como El Salvador, éste sería muy distinto al que aparece en los manuales al uso.

$\mathrm{Al}$ atender a ciertos indicadores sociodemográficos destacados o mayoritarios, el tipo ideal salvadoreño o el "habitante" de un hipotético manual de Psicología del desarrollo salvadoreño, debería tener alguna de las siguientes características ${ }^{6}$ : viviría en sectores urbanos (62\%), tendría menos de 30 años (56 \%), sería una mujer ${ }^{7}$, contaría con una escolaridad promedio urbana de casi ocho años; un ingreso familiar promedio urbano de $\$ 661.90$ (el ingreso familiar promedio nacional es de $\$ 556.16$ ); y habitaría en un hogar compuesto por un estimado de cuatro personas (DIGESTYC, 2014). Cabría añadir que, con bastante probabilidad, estaría subempleado o tendría un empleo de mala calidad (Orellana, 2012; PNUD, 2008) ${ }^{8}$, por lo que, a largo plazo, se vería imposibilitado para acceder a una pensión digna (Córdova et al., 2010); es religioso o creyente (Instituto Universitario de Opinión Pública [IUDOP], 2009); su familia es nuclear (38 \%) o extensa (32 \%), usualmente con hijos y con familiares en el extranjero o se ve inmersa en importantes dinámicas trasnacionales (Benítez, 2011; Fundación Salvadoreña para el Desarrollo Económico y Social [FUSADES] y Fondo de las Naciones Unidas para la Infancia [UNICEF], 2015; Gaborit, et al., 2015, 2016; Orellana, 2016; PNUD, 2013); por último, muy probablemente su hogar experimentaría pobreza en términos multidimensionales (62\%) (Secretaría Técnica de la Presidencia de la República [STPP] \& Ministerio de Economía [MINEC]-DIGESTYC, 2015).

\footnotetext{
${ }^{6}$ Son características necesariamente imprecisas y provisionales porque tras cada categoría aparecen subdivisiones o discrepancias entre taxonomías, etiquetas y fuentes. Lo importante es el ejercicio en sí mismo de construcción grosso modo del habitante salvadoreño promedio o mayoritario que las fuentes disponibles permiten elaborar.

${ }^{7}$ Por poco: en el país las mujeres representan el $53 \%$ de la población y el restante 47 \% corresponde a los hombres. En promedio se considera que en el país existen 90 hombres por cada 100 mujeres.

${ }^{8}$ En este recuento, he evitado con toda intención incluir los indicadores del Área Metropolitana de San Salvador (AMSS). Esta región geográfico-administrativa es la que suelen destacar los informes, en buena medida, porque cuenta con el más alto desarrollo socioeconómico y es fundamentalmente urbana. No obstante, según el informe de la DIGESTYC, en el AMSS habita apenas algo más de la cuarta parte de la población salvadoreña $(28 \%)$.

9 Según STPP y MINEC-DIGESTYC (2015), un hogar presentaría pobreza multidimensional al cumplir siete criterios asociados a cinco dimensiones sociales (educación, vivienda, trabajo, salud y calidad del hábitat): se supone que este es el caso de "únicamente" poco más de un tercio de los hogares salvadoreños (35\%). Sin embargo, según el mismo informe, más allá de esta convención adoptada (los siete criterios), el promedio de privaciones de los hogares salvadoreños es de casi seis, lo que equivale a la proporción de hogares reportada arriba (62 \% ). Una discusión crítica de la pobreza, la exclusión y la desigualdad aplicada
} 
Como es de esperar, el escenario es mucho más complicado y apremiante si del área urbana pasamos a la situación concreta de las zonas rurales.

Los rasgos y los correlatos materiales y simbólicos de una sociedad inhóspita constituyen verdaderos desafíos para cualquier reflexión sobre el decurso del ciclo vital de los miembros de tal sociedad. Tanto las expresiones no normativas como el actor social que se dibujan, en el apurado esbozo anterior, pierden en "glamour" lo que ganan en fidelidad con la realidad cotidiana que constituyen. Tal vez desactivan teorías hechas, pero abren posibilidades inéditas para pensar el desarrollo humano. Esquivan la aplicación mecánica de categorías, mientras impelen a agudizar la mirada, a forzar y sacudir el lenguaje que las nombra. Justo como debería ocurrir con el posicionamiento de quien investiga y reflexiona situado e inmerso en ese mismo escenario inhóspito común.

\section{Conclusión: La necesidad de un conocimiento nuevo a través de una mirada nueva}

Estas reflexiones no pretenden ser una suerte de cruzada abolicionista contra la Psicología del desarrollo. Se propone un replanteo de contenido, no de forma. Sería absurdo claudicar y prescindir del acervo de conocimientos de la disciplina o de sus reconocidas categorías analíticas (etapas, crisis, hitos, procesos, etc.). Pero no menos absurdo es pretender que tales recursos son inamovibles o que se manifiestan libres de presupuestos ideológicos o de forma universal, como ojalá se haya podido ilustrar a propósito de la crítica hecha a los llamados procesos normativos y no normativos. La apuesta es por una Psicología del desarrollo situada y pertinente, cuyos fundamentos y sus propuestas respondan a una dialéctica de cuestionamiento constante entre sus propios planteamientos y la realidad que objetivamente le circunda. Aquí, se sostiene que tal realidad así como el desarrollo psicológico que en ella puede tener lugar, podría responder - es al menos el caso salvadoreño- a las características de una sociedad inhóspita antes que a construcciones abstraccionistas o importadas de forma acrítica. $\mathrm{Si}$ bajo tales condiciones adversas se identifican ciclos de vida predominantemente desventajosos y que, por tanto, adquieren un cariz normativo para las personas, una psicología del desarrollo crítica tendrá que decir algo al respecto. Para ello, echará mano de las herramientas de que dispone, las adecuará o creará unas nuevas, mientras también problematiza e increpa el carácter deshumanizante de tales condiciones. Lo que no es admisible es la cómoda complicidad silente de la academia al preferir crear o importar, con mayor o menor conciencia de ello, realidades supuestamente homeostáticas y constantes y que, además, esto le parezca tan normal como normativo. Es necesario reintroducir la realidad en nuestras ideas (por muy fea que sea) antes que confundamos las unas con la otra. De manera coloquial, la desconexión de la realidad es entendida como locura. No puede ser que la ciencia social y la ciencia psicológica en particular de nuestros países prescinda de su cordura a conveniencia, ni que a base de negación para enfrentar sus propias falencias se resista a pensar realidades violentas o quebradas por la desigualdad que, sin embargo, son las que vertebran la cotidianidad de la gran mayoría de la población. Pero, entonces, ¿qué hacer? Algunas reflexiones para comenzar andar y a hacer camino se ofrecen a continuación.

Para la construcción de una Psicología del desarrollo en nuestros países -y me temo que para casi cualquier rama del saber psicológico también-, sigue siendo necesario establecer una nueva epistemología y una nueva praxis que asuma una posición de enunciación clara (Martín-Baró, 1986); es decir, elaborar un nuevo conocimiento, sobre todo poner en marcha nuevas vías de indagación, problematización y construcción. Una epistemología que se interese y pregunte, que se sirva del conocimiento dominante, pero simultáneamente a contrapelo, cómo se vive cuando se malvive o se sobrevive, cuando la existencia usual para muchos es sortear una

al caso centroamericano puede encontrarse en el libro de Juan Pablo Pérez-Sáinz que ha sido citado antes. 


\section{Orellana}

realidad cuesta arriba. Por ejemplo: ¿cómo es el ciclo vital de la niña-madre de otra(s) niña(s); del joven cuyas posibilidades materiales de existencia lo convierten en carne de "Nini" llegar a fin de mes? ¿Cuáles presiones de género y cuáles procesos de etiquetamiento y de patologización sufren las mujeres debido a procesos "normados por la edad" como el binomio menopausia/climaterio? ¿Qué ocurre en la mayoritaria vejez sin pensiones, júbilo ni jubilación del anciano -o la anciana con una expectativa de vida mayor- que asiste al fin de sus días entre la desesperación y el abandono? Si de entre la tenebrosa estadística de los 20 homicidios diarios que actualmente se cobra la violencia en El Salvador, a una madre le toca -cuando puede- dar sepultura a más de un hijo, en más de una ocasión de manera simultánea debido a las recurrentes masacres, ¿qué ocurre con la eficacia simbólica de los ritos, con las consabidas etapas secuenciales del duelo?.

Según Burman (1997, 2015), el camino por seguir para la elaboración de una Psicología del desarrolo crítica pasa por asumir posturas construccionistas, deconstruccionistas y hasta "antidesarrollo"; es decir, perspectivas que den cuenta de lo singular antes que de nociones cargadas de regularidades naturales o que, lejos de guardar lealtad a modelos explicativos unívocos, procuren exponer sus sesgos ideológicos y sus limitaciones para entonces generar un conocimiento más pertinente. También, para la autora, este esfuerzo conllevaría otras estrategias, a saber: a) procurar la representación cultural o la contextualización de la condición de niñez (por ejemplo, las peculiaridades de la niñez migrante irregular, en Orellana, 2014); b) colocar el foco de atención en la vida cotidiana y asumir la existencia de múltiples formas de desarrollo psicológico. Debe recordarse que el "ciclo vital", etiqueta que traduce una idea de proceso experiencial uniforme, está, en realidad, mediado por aspectos como la clase social, el género o la etnia (ver Baltes, 1987); c) el tratamiento teórico y político tanto de la relación como de la autonomía de la madre y los niños y las niñas, así como las formas de control social que se esconden tras enfoques supuestamente elaborados en favor suyo y para "darles voz".; d) vigilancia sobre modelos homogeneizadores de conocimiento y de desarrollo político-económico que dinamizan conocimientos propios de psicología del desarrollo humano; e) evitar toda forma de psicologismo que fortalezca visiones reduccionistas de problemas políticos (e.g. ante la maternidad adolescente, sugerir programas de "estimulación infantil"; ante el desempleo femenino, "fortalecer la autoestima de la mujer"; ante los crecientes "Ninis", ofrecer charlas de "motivación personal" o de "emprendedurismo"); por último, f) el acercamiento estratégico a campos de conocimiento distintos a la psicología que pueden enriquecer dicha tarea (e.g. antropología, teoría cultural, teoría postcolonial, teoría feminista, etc.).

Una dirección prometedora en este sentido parece marcar la llamada Psicología del Ciclo Vital (Psychology of the Life Span o Life-Span Developmental Psychology), la cual supera nominalmente la carga biologista de la etiqueta de la psicología "evolutiva", o la de progreso mecánico y economicista de la psicología del "desarrollo". La Psicología del ciclo vital ya comprendería un salto cualitativo respecto de la Psicología del desarrollo al uso,

\footnotetext{
${ }^{10}$ Se trata de jóvenes que ni estudian ni trabajan. El último informe del Banco Mundial sobre los Ninis en Latinoamérica (De Hoyos, Rogers y Székely, 2016) sostiene que existirían unos 20 millones de jóvenes con estas características en la región. Para el caso de Centroamérica, tanto en Honduras como en El Salvador, arriba del 25 \% de jóvenes calificarían como ninis. Esta condición masiva de desocupación se ve alimentada por aspectos propios de una sociedad inhóspita (baja escolaridad, adultización prematura, empleos precarios, etc.). De fondo, este es un escenario confirmatorio de la incapacidad de los Estados para aprovechar el bono demográfico de adultos jóvenes que como capital humano y económico podrían incidir en las condiciones de productividad y de bienestar de nuestros países (sobre la transición demográfica en Centroamérica y la evolución del bono demográfico ver los informes del PEN, 2011, 2016).
} 
al enfatizar aspectos como la importancia de la contextualización, la fluidez de la existencia y el interjuego entre las ganancias y las pérdidas antes que la rigidez de las etapas y de la edad, o por concebir la vida humana en sus distintos momentos como una oportunidad de aprendizaje y crecimiento constante antes que como un proceso ininterrumpido o como un declive inevitable (Arranz Freijo, 1998; Baltes, 1987; Dulcey-Ruiz, 2010; DulceyRuiz \& Uribe Valdivieso, 2002). Una buena dirección, sin duda, pero que debe evitar el mero cambio nominal o la réplica del vicio mimético de contenido en el que ya se incurre con la Psicología del desarrollo dominante.

Suele decirse que las circunstancias adversas sacan lo peor y lo mejor de los seres humanos. Lo propio ocurre con la producción de conocimiento. Resistir a la tentación de la copia mecánica y descontextualizada de modelos obliga, además, a reacomodos prácticos y a reinvenciones epistemológicas. En lo que a reacomodos prácticos se refiere, cabe decir que la experiencia docente de la Psicología del desarrollo, por todo lo apuntado, conduce al enriquecedor y constante ejercicio de contextualización, de contraste y de crítica. De la precariedad nace la inventiva, la observación acuciante, la ironía como dispositivo de toma de distancia de condiciones que por óptimas aparecen como lejanas. Desencantos, frustraciones e indignación constante aparte, una sociedad inhóspita constituye un antídoto amargo que se toma a voluntad para combatir el apoltronamiento intelectual. También, las buenas intenciones en la enseñanza pueden perderse en la fugacidad de la sesión de clase (recuérdese el confinamiento epistemológico). De ahí que sea menester buscar las reinvenciones epistémicas aludidas.

En un recuento rápido de la evolución histórica de la psicología en Latinoamérica, Ardila (1998) establece que, desde 1950, la psicología de la región se vio enfrascada en el reconocimiento de la insuficiencia y la insatisfacción con los modelos foráneos y, al mismo tiempo, en la construcción de una filosofia propia en un esfuerzo de "búsqueda de autenticidad" (p. 25). Si bien hay que asumir que el desarrollo de la psicología en la región como la región misma, distan de ser homogéneos, es llamativa esta búsqueda de sí, de autenticidad y de filosofía original, porque Latinoamérica no es ajena a las sociedades inhóspitas, como la filosofía no es ajena a la ignorancia, todo lo contrario. Conviene hacer nuestra la reflexión de Savater (2008) cuando sostiene que la filosofía no sirve para salir de dudas sino para entrar en ellas y que solo se ve orillado a filosofar - es decir, obligado a pensar, a crear, a escudriñar, a criticar, a proponer--aquel que se ve en problemas, quien no la ha tenido fácil y se ve abocado a la necesidad de pensar fuera de lo establecido. Buscar la filosofía propia no implica volver al tronco común de donde una vez se desprendió la ciencia. Más bien, supone la elaboración de paradigmas y teorías consecuentes con el contexto de interés. En el contexto latinoamericano y de países como El Salvador, esto remite a abrazar la hibridez que se cristalizaría entre el conocimiento dominante y el incipiente conocimiento subalterno local (lo que incluye el conocimiento popular).

Este esfuerzo filosófico-epistémico, puede encontrar mucho de su lógica procedimental como de su impulso fundamental en el llamado Pensamiento posabismal de Santos (2010). A grandes rasgos, el pensamiento posabismal será aquel que supera la división maniquea (pensamiento abismal) entre saberes visibles e invisibles, excluyentes y excluidos. Se trata de contrarrestar formas de conocimiento que generan abismos que separan a humanos y no humanos, a hegemonías y subalternidades, que elimina la dialéctica y la copresencia del conocimiento. Para Santos, el pensamiento posabismal constituiría una ecología de saberes que reconoce que se debe pensar desde "el otro lado de la línea" (donde habita el otro usualmente invisibilizado), que el conocimiento es "interconocimiento" o que el saber dominante debe ser empleado 


\section{Orellana}

de formas contrahegemónicas ${ }^{11}$. Este guiño a la sociología del conocimiento nos sitúa frente a otra de las reinvenciones epistemológicas que la construcción de una Psicología del desarrollo nos revela: asumir la paradoja de la adopción de una perspectiva multidisciplinaria si lo que se busca es acrecentar el campo específico de conocimiento.

Parte de la miseria epistemológica de la ciencia social, de la Psicología en general y de la Psicología del desarrollo en particular, al menos por estos lados, radica en su talante pueblerino. Este se manifestaría en la tendencia a seguir y maravillarse por lo que se hace fuera, también a través de la incapacidad o la renuencia del saber académico para entrar en diálogo con otras disciplinas (o con otras áreas de la misma psicología) para generar conocimiento más rico y de mayor alcance. Pero, una Psicología del desarrollo crítica u orientada a consolidarse, digamos, al menos, como una Psicología del ciclo vital, requiere ver más allá de los linderos de la propia disciplina psicológica, especialmente por la tendencia dominante de esta al reduccionismo microsocial (biologista y psicologista) y porque, por definición, el desarrollo humano necesita una mirada amplia que no puede agotarse en una perspectiva única. Menos aún frente a realidades tan complejas y que ponen a prueba la capacidad de representación y problematización como ocurre con una sociedad inhóspita.

Sucede, sin embargo, que este esfuerzo requiere romper inercias con raíces muy profundas, en el gremio de la Psicología,o también en la academia latinoamericana en general. Al ejemplo, Mu y Pereyra-Rojas (2015) constatan en el marco de los Estudios Latinoamericanos, que los académicos de la región y que viven en ella, son los que cuentan con menos producción académica en comparación con aquellos del mundo anglohablante (Estados Unidos y Reino Unido). Esto se debería, entre otros aspectos, a la autodefinición de los primeros como "agentes de cambio". Esta adscripción identitaria sería el fruto de experiencias personales en contextos sociales precarios (guerra, ambientes politizados, exclusión, socialización religiosa), el seguimiento utópico de un mandato ético de "transformar" las condiciones de vida que les rodean y por realizar su labor en contextos académico-institucionales restrictivos, entre otros aspectos. Dicho de otra forma, mucho de Latinoamérica como objeto de estudio, emerge de la visión de académicos no latinoamericanos que tampoco residen en la región. Por su lado, Zych, Buela-Casal, Paz Bermúdez y Cierra (2013) muestran que, a nivel mundial, las psicólogas y los psicólogos se conciben, a sí mismos, fundamentalmente, como "una profesión de la salud". Esto trae como consecuencias encontrar paralelismos entre la Medicina y la Psicología (especialmente los psicólogos de los Estados Unidos participantes en el estudio), que el campo de la psicología clínica sea identificado con la razón de ser y el quehacer de la psicología como profesión y como ciencia, o sostener la aspiración global por unificar tratamientos para personas de distintos orígenes sociales y geográficos.

En esta tesitura, ¿qué clase de cambio puede propiciar y cuánta agencia real puede tener un profesional que hace sin conocer o que importa conocimiento o que no construye brújulas teóricas contextualizadas para, supuestamente, transformar la misma realidad que le rodea? ¿Qué margen de crecimiento crítico puede adoptar la psicología en su eterna identificación con uno de los modelos -el médico-más reduccionistas en términos epistemológicos? ¿Por qué concebirse sólo como una "profesión de la salud" y no también como una ciencia social cuando el espectro de alcance y de reflexión de la ciencia psicológica se adentra

${ }^{11}$ Esta aproximación es convergente con lo que Burman (2015) denomina Psicología del desarrollo deconstruccionista. 
en niveles de análisis, cuenta con categorías, modelos y herramientas para dar cuenta de lo social? ${ }^{12}$ ¿Qué dice una profesión y una ciencia de sí misma cuando demuestra tanta estrechez de horizontes de acción y de conocimiento? Sin duda, las interrogantes, al respecto, podrían multiplicarse.

Los vicios y los riesgos del activismo, el formalismo administrativo, la visión parroquial del conocimiento o la idealización del saber venido de fuera, la estrechez de miras y hasta los complejos de inferioridad de la Psicología clínica respecto a la medicina, hablan del rezago generalizado en el que la Psicología se encuentra inmersa por estos lados. Solo podrán ser contrarrestados con un replanteo del quehacer de los mismos profesionales donde, al menos, las universidades deben ser señaladas como corresponsables por perpetuar tales vicios así como los "roles tradicionales de gremio" en sus escuelas. El contexto de las sociedades inhóspitas y la discusión en torno a los problemas particulares de la Psicología del desarrollo han servido, también, para poner sobre la mesa estas discusiones de más amplio alcance. A las sugerencias generales que arriba se han ofrecido, cabría añadir, para concluir, que la construcción de una Psicología del desarrollo crítica, o al menos la transición real a una Psicología del ciclo vital, pasa primero por considerar el subdesarrollo de la psicología misma, así como la reflexión previa o simultánea sobre una psicología del subdesarrollo. Cualquier otro camino solo daría nuevos aires a las insuficiencias revisadas. Dar la espalda a estas tareas y desafíos supondría desviar evasivamente la mirada de los múltiples y los complejos ciclos vitales y de los rostros que se asoman en los intersticios de una sociedad inhóspita que hace tiempo mira fijamente y expectante a los ojos de los "profesionales de la salud" y sus siempre renovados problemas de miopía, de crisis de identidad y de inmadurez científica.

\footnotetext{
${ }^{12}$ En El Salvador, la profesión de la Psicología está adscrita al Consejo Superior de Salud Pública (CSSP). Mientras escribo estas líneas, la Junta de Vigilancia de la Profesión en Psicología se encuentra en un proceso de actualización de "sellos y datos" de los y las profesionales del gremio. En un país donde el imaginario social aún identifica la Psicología clínica con la Psicología en general y en el marco de la discusión que nos ocupa, es llamativo constatar que: el distintivo de la profesión sea un artilugio performativo (un sello) distintivo de la medicina o de la psiquiatría; que sea imperativo obtener tal cosa asumiendo erróneamente que el quehacer de la Psicología toda se reduce a certificar estados de salud; que en los formularios de registro se asuma además que quien lo llena cuenta con "clínica particular", pero no se considere la posibilidad de contar con un doctorado; o que en el sitio web gubernamental donde aparecen los "profesionales de la salud" del país quienes contamos con el grado de psicología aparezcamos en una carpeta o directorio identificado bajo la etiqueta de "doctors". Quizás, somos mucha profesión y muy poca ciencia, y la movilidad social siempre ha sido más apremiante que la producción de conocimiento, a pesar de las serias implicaciones que la ignorancia o la descontextualización pueden acarrear para el ejercicio cotidiano de la profesión. El mayor estatus cultural del campo "clínico" -léase sanitario, paramédico, correctivo, pedagógico, terapéutico, peritaje- se explica en buena medida por el cumplimiento aspiracional autómatico de poder ser propietario de "una clínica o consultorio privado", el prestigio vicario, la consabida derivación y la dependencia formativa histórica de la medicina (ver Calderón de Orellana, 2009). Cabe destacar que tal estatus no proviene de una mayor o mejor formación o entrenamiento de la psicología clínica respecto a otros campos del saber psicológico: en el país no se exige una especialización que acredite como especialista en la atención clínica de personas como sí se hace en otros lados. Las implicaciones son muy graves: abuso desde la inexperiencia y el sentido común, etiquetamiento, revictimización y empeoramiento de problemas, aplicación inexperta, ilegal y acrítica de pruebas, patologización de la vida cotidiana, charlatanería y vulgarización del quehacer psicológico, proliferación de "consejeros" de todo tipo (pastores/as, aplicadores de terapias "alternativas", etc.), entre otras cosas y muchas de ellas combinadas entre sí.
} 


\section{Orellana}

\section{Referencias}

Ardila, R. (1998). La Psicología en América Latina. Pasado, presente y futuro (2a ed.). México, D. F.: Siglo Veintiuno Editores.

Arranz-Freijo, E. (1998). Modelos del Desarrollo psicológico humano (2a ed.). Bilbao, España: Universidad del País Vasco.

Baltes, P. (1987). Theoretical Propositions of Life-Span Developmental Psychology: On the Dynamics Between Growth and Decline. Developmental Psychology, 23(5), 611-626.

Benítez, J. L. (2011). La comunicación trasnacional de las e-familias migrantes. San Salvador, El Salvador: PNUD y UCA.

Burman, E. (1997). Developmental Psychology and its Discontents. En D. Fox \& I. Prilleltensky (Eds.), Critical Psychology. An Introduction (pp. 134-149). Londres, Inglaterra: Sage Publications.

Burman, E. (2015). Developmental Psychology. The turn to deconstruction. En I. Parker (Ed.), Handbook of Critical Psychology (pp. 70-78). Londres, Inglaterra y Nueva York, EE. UU.: Routledge.

Calderón de Orellana, L. (2009). Historia de la Psicología en El Salvador 1928-2006 (2a ed.). San Salvador, El Salvador: Imprenta Criterio.

Córdova, R., Burgos, D., Tablas, V., \& Rodríguez F. (2010). Las tendencias demográficas de la población adulta mayor y sus implicaciones para las politicas públicas en materia de seguridad social. San Salvador, El Salvador: FUNDAUNGO y UNFPA.

Craig, G. (2001). Desarrollo Psicológico (8a ed.). México, D. F.: Prentice Hall.

De Hoyos, R., Rogers, H., \& Székely, M. (2016). Ninis en América Latina. 20 millones de jóvenes en busca de oportunidades. Washington, D.C., EE.UU.: Banco Mundial. Recuperado de https://openknowledge.worldbank.org/ handle/10986/22349

Dirección General de Estadística y Censos (DIGESTYC). (2014). Encuesta de Hogares de Propósitos Múltiples 2013. Recuperado de http://www.digestyc.gob.sv/index.php/temas/des/ehpm/publicaciones-ehpm.html

Dulcey-Ruiz, E. (2010). Psicología social del envejecimiento y perspectiva del transcurso de la vida: consideraciones críticas. Revista Colombiana de Psicología, 19(2), 207-224.

Dulcey-Ruiz, E., \& Uribe Valdivieso, C. (2002). Psicología del Ciclo vital: hacia una visión comprehensiva de la vida humana. Revista Latinoamericana de Psicología, 34(1-2), 17-27.

Feldman, R. (2007). Desarrollo psicológico a través de la vida (4a ed.). México, D.F.: Pearson.

Fernández-Lópiz, E. (2000). Explicaciones sobre el Desarrollo humano. Madrid, España: Ediciones Pirámide.

Fundación Salvadoreña para el Desarrollo Económico y Social (FUSADES) \& Fondo de las Naciones Unidas para la Infancia (UNICEF). (2015). Una mirada a las familias salvadoreñas: sus transformaciones y desafios desde la óptica de las políticas sociales con enfoque hacia la niñez. Recuperado de http://fusades.org/node/6695

Gaborit, M., Zetino, M., Orellana, C. I., \& Brioso, L. (2015). El Salvador. En K. Musalo, L. Frydman, \& P. Ceriani (Coords. y Eds.), Niñez y migración en Centro y Norte América: causas, políticas, prácticas y desafios (pp.167-209). Recuperado de http://cgrs.uchastings.edu/Ninez-Migracion-DerechosHumanos 
El DESAFí́ DE CONSTRUIR UNA PSICOLOGÍA DEL DESARROLlo CRÍTICA EN SOCIEDADES INHÓSPITAS

Gaborit, M., Zetino Duarte, M., Orellana, C.I., Brioso, L., Rodríguez Burgos, M., \& Avelar, D. (2016). Atrapados en la tela de araña. La migración irregular de niñas y niños salvadoreños hacia los Estados Unidos. San Salvador, El Salvador: Talleres Gráficos UCA.

Gutiérrez, R. (2013). Evaluación de factores psicosociales de riesgo de protección de violencia juvenil en jóvenes. Recuperado de http://www.utec.edu.sv/Inicio/Publicaciones/Coleccion-Investigaciones/ Evaluacion-de-factores-psicosociales-de-riesgo-y-proteccion-de-violencia-juvenil-en-El-Salvador

Ibáñez, T. (2001). Psicología Social Construccionista (2a ed.). México, D.F.: Universidad de Guadalajara.

Instituto Universitario de Opinión Pública (IUDOP). (2009). Encuesta sobre la religión para las y los salvadoreños. Recuperado de http://www.uca.edu.sv/publica/iudop/Web/2009/informe122.pdf

Lefrancois, G. (2001). El Ciclo de la Vida (6a ed.). México, D.F.: Thomson.

Martín-Baró, I. (1986). Hacia una Psicología de la Liberación. Boletín de Psicología, 22, 219-231.

Ministerio de Salud (MINSAL) \& Instituto Nacional de Salud (INS) (2015). Maternidad y Unión en niñas y adolescentes: Consecuencias en la vulneración de sus derechos. El Salvador 2015. Primera lectura de datos. Recuperado de https://www.salud.gob.sv/archivos/comunicaciones/archivos comunicados2015/pdf/maternidad union ninas y adolescentes 1ra lectura datos.pdf

Mu, E., \& Pereyra-Rojas, M. (2015). Impact on Society versus Impact on Knowledge. Why Latin American Scholars Do Not Participate in Latin American Studies. Latin American Research Review, 50(2), 216-238.

Orellana, C. I. (2012). Exclusión, crisis del mundo del trabajo y precariedad: A vueltas con el tema de la ciudadanía. Estudios Centroamericanos (ECA), 67(729), 229-258.

Orellana, C. I. (2014). Migración irregular de niños y de niñas: Ejercicio y búsqueda de ciudadanía desde la invisibilidad. Estudios Centroamericanos (ECA), 69(739), 345-374.

Orellana, C.I. (2016). Pensando en marcharse: niñas y niños potenciales migrantes ante la consideración de la migración irregular. En M. Gaborit, M. Zetino Duarte, C. I. Orellana, L. Brioso, M. Rodríguez Burgos y D. Avelar, Atrapados en la tela de araña. La migración irregular de niñas y niños salvadoreños hacia los Estados Unidos (pp. 122-177). San Salvador, El Salvador: Talleres Gráficos UCA.

Papalia, D., \& Feldman, R. (2012). Desarrollo Humano (12a ed.). México, D. F.: McGraw-Hill/Interamericana Editores.

Pérez-Sáinz, J. P., Segura Carmona, R., \& Fernández Montero, D. (2012). La exclusión social en Centroamérica al inicio del Siglo XXI. Una interpretación cuantitativa. En J. P. Pérez-Sáinz (Ed.), Sociedades Fracturadas. La exclusión social en Centroamérica (pp. 49-110). San José, Costa Rica: Flacso-Costa Rica. Recuperado de http://www.clacso.org.ar/ libreria cm/archivos/pdf 195.pdf

Programa de las Naciones Unidas para el Desarrollo (PNUD). (2008). Informe sobre Desarrollo humano El Salvador $2007-$ 2008. El empleo en uno de los pueblos más trabajadores del mundo. San Salvador, El Salvador: Autor.

Programa de las Naciones Unidas para el Desarrollo (PNUD). (2013). Informe sobre Desarrollo humano El Salvador 2013. Imaginar un nuevo país. Hacerlo posible. San Salvador, El Salvador: Autor. 


\section{Orellana}

Programa de las Naciones Unidas para el Desarrollo (PNUD). (2014). Informe sobre Desarrollo humano 2014. Sostener el progreso humano: reducir vulnerabilidades y construir resiliencia. Nueva York, EE.UU.: Autor.

Programa Estado de la Nación (PEN). (2011). Cuarto informe Estado de la región en Desarrollo humano sostenible. San José, Costa Rica: Programa Estado de la Nación.

Programa Estado de la Nación (PEN). (2016). Quinto informe Estado de la región en Desarrollo humano sostenible. San José, Costa Rica: Programa Estado de la Nación.

Rice, P. (1997). Desarrollo Humano (2a ed.). México, D.F.: Pearson-Prentice Hall.

Santacruz, M., \& Carranza, M. (2009). Encuesta Nacional de Juventud. Análisis de resultados. San Salvador, El Salvador: IUDOP.

Santos, B. (2010). Descolonizar el poder, reinventar el poder. Montevideo, Uruguay: Ediciones Trilce-Extensión universitaria.

Savater, F. (2008). La aventura del pensamiento. Buenos Aires, Argentina: Editorial Sudamericana.

Shaffer, D., \& Kipp, K. (2010). Developmental Psychology. Childhood and Adolescence (8a ed.). Belmont, CA., EE.UU.: Wadsworth Cengage Learning.

Secretaría Técnica de la Presidencia de la República (STPP) \& Ministerio de Economía (MINEC)-DIGESTYC (2015). Medición multidimensional de la pobreza. El Salvador. San Salvador, El Salvador: Secretaría Técnica y de Planificación de la Presidencia y Ministerio de Economía-DIGESTIC.

Tapia Valladares, J., Carmiol Barbosa, A. M., \& Rosabal Coto, M. (2012). La Psicología del desarrollo en Costa Rica: Alcances y perspectivas futuras. Revista Costarricense de Psicología, 31(1-2), 101-121. Recuperado de http:// www.rcps-cr.org/openjournal/index.php/RCPs/article/view/11/10

Zych, I., Buela-Casal, G., Bermúdez, M. P., \& Sierra, J. C. (2013). Is Psychology a Health Profession? An Opinion of a Sample of Psychologists Worldwide. Universitas Psychologica, 12(1), 157-171. Recuperado de http://revistas. javeriana.edu.co/index.php/revPsycho/article/view/1640/3971

Recibido: 18 de febrero de 2016 Revisión recibida: 29 de Agosto de 2016

Aceptado: 07 de octubre de 2016

\section{Sobre el autor:}

Carlos Iván Orellana es Doctor en Ciencias Sociales por la Facultad Latinoamericana de Ciencias Sociales (FLACSOCentroamérica). Trabaja como investigador y profesor del Departamento de Psicología y de Salud Pública de la Universidad Centroamericana José Simeón Cañas (El Salvador). Desde una perspectiva multidisciplinaria, su trabajo e interés académico actual se concentra en el estudio teórico y empírico de fenómenos como la violencia y la inseguridad, el autoritarismo, los crímenes de odio y la migración irregular de niños y de niñas. 